\title{
OPTIMISATION OF CONCRETE SLEEPER DESIGN FOR HEAVY HAUL OPERATED RAILWAY TRACK BY NON-LINEAR FINITE ELEMENT AND EMPIRICAL STUDIES
}

\author{
RALPH ZHANG ${ }^{1,2}$, HELEN WU ${ }^{1}$ \& RICHARD YANG ${ }^{1}$ \\ ${ }^{1}$ Western Sydney University, Australia \\ ${ }^{2}$ Sydney Trains, Australia
}

\begin{abstract}
Concrete sleeper is one of the major railway track components and it has been successfully applied by the railway authorities around the world for over 60 years. As part of Australian railway's North South Corridor track strengthening strategy in 2005 , the new type of medium duty and heavy duty prestressed concrete sleepers have been designed based on the outcomes of a new innovation of the track condition and technical design parameters. As the process of technical evaluation and design fine tune of the newly designed concrete sleepers, a nonlinear finite element analysis model is created. This model includes the 3-dimensional concrete sleeper, steel tendons/reinforcements in the concrete sleeper and ballast layer underneath the sleeper. In this paper, the outcomes from the innovation of track condition and the determination of design parameters are presented. Moreover, some technical suggestions based on results from the numerical study and empirical studies are addressed.
\end{abstract}

Keywords: concrete sleeper, heavy haul, finite element, empirical study.

\section{INTRODUCTION}

Concrete sleeper is one of the major track elements which is design to carry out the functions of supporting of rail, spread wheel loads to ballast, hold rails to gauge and inclination, transmit lateral and longitudinal forces, insulate rails electrically, and provide a base for rail seats and fastenings.

For over 60 years, prestressed concrete sleepers have shown its successful application around the world and have almost completely replaced timber sleepers in many countries. Their technical superiority is based not only on the greater load-bearing capability, but also, and especially, on their longer life-cycles. Their service lives lie between 30 and 60 years, depending on the type of loads applied.

As part of the largest Australian railway authority's track strengthening strategy (which is linked up with all of the three capital cities - Melbourne, Sydney and Brisbane on the east coast of Australia) in 2005, the new type of medium duty and heavy duty prestressed concrete sleepers have been designed based on the outcomes of a new innovation of the track condition and technical design parameters such as dynamic factor of wheel load, sleeper spacings, etc.

\section{PERFORMANCE SPECIFICATION}

The new designed concrete sleeper is a type of medium duty concrete sleeper for the standard gauge tracks which are operating the $25 \mathrm{t}$ axle load freight train predominated traffic at speed of $80 \mathrm{~km} / \mathrm{h}$. It is considering installation of 1500 sleepers $/ \mathrm{km}$, which equates to a $667 \mathrm{~mm}$ sleeper spacing. The length of the sleeper is $2500 \mathrm{~mm}$ and assembly with resilient fastening systems. As described in section 5.4 of AS 1085.14 (Australian Standard [1]), Design Parameters, an impact factor of 2.04 is recommended for the design of medium duty concrete sleeper. Hence, the impact factor 2.04 is utilised. 
Within Australia, based on more than half centuries' heavy haul revenue, it has been noted that the track maintenance works (tamping, etc.) are directly affected by the contact stress between underneath of concrete sleeper and ballast. A great maintenance benefits follow from the reducing of ballast contact stresses. A key issue in track design is the design contact stress imposed by a sleeper on ballast. AS 1085.14 suggest this should be a maximum of $750 \mathrm{kPa}$. Substituting $667 \mathrm{~mm}$ spacing and the purposed $290 \mathrm{~mm}$ base width would reduce the contact pressure to $437 \mathrm{kPa}$, well below the recommended $500 \mathrm{kPa}$ maximum.

\section{CURRENT DESIGN PRACTICES}

One of the design practices of concrete sleeper design since 1990s is the $6 \mathrm{~mm}-10 \mathrm{~mm}$ steel tendon instead of the multi-ply steel wires/strands $(\Phi 3 \times 3 \mathrm{~mm}$ or 7 ply strands). Extensive research and testing carried out by German, Chinese and Swedish railway authorities has shown that the single reinforcing steel bar can provide better performance than the ply strands or wires. Two types of concrete crack - longitudinal crack between a pair of rail fasteners and sleeper end crack were believed to have relationship with pre-tensioned ply steel wires.

According to a research which was carried out by the Chinese Railway Society in 1990s' to 2003 and researched by the Swedish railway have shown, the most severe cracks that directly results concrete sleeper failure were not the cracks that caused by the positive bending moment from wheel load at rail seats, and as well as the negative bending moment of ballast reaction at the centre of the concrete sleeper. The most frequent and major serious crack type is "the longitudinal crack between/around the two steel shoulders at a rail seat that are used to fix one rail", as shown in Fig. 1.

A long term of in-field investigation of Chinese Type S-II concrete sleeper (designed and manufactured in 1980s') which was carried out by the Chinese Railway Academy has shown that the major type of concrete crack is the longitudinal crack between the two steel shoulders, which is equal to $33.8 \%$ of the overall numbers of cracked ineffective concrete sleepers. This type of crack usually starts from the shoulders and progresses to the centre and end of the concrete sleeper. This problem has also been reported by some German and Swedish innovation papers (Gustavson [2] and Vincent [3]). An interesting finding from this research was that with the same installation and maintenance situation, the J-I concrete sleeper which utilises the single steel reinforcement, provided a much better durability performance than the S-II concrete sleeper which utilised eleven $\Phi 3 \times 3 \mathrm{~mm}$ steel wires. Although the J-I sleeper was designed as a lower class to the S-II sleeper, the number of damages, especially the longitudinal cracks were decreased dramatically.

A research carried out by the Tsinghua University of China (Wang and Bai [4]) can be used to explain another type of longitudinal crack - the sleeper end crack which occurred with the pre-tensioned high strength ply wires concrete sleeper. For the pre-tensioned high strength ply wire concrete beams, if the initial tensioning force is high, beam end crack will

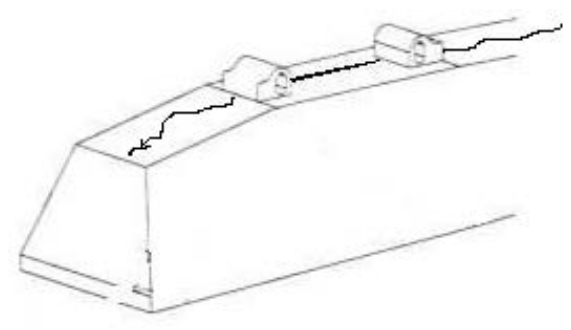

Figure 1: Longitudinal crack between the two steel shoulders at rail seat. 
occur by the tension stress $\sigma_{Y}$ at the location along the height of concrete beam and apart from the location of the tensioned steel ply wires near the neutral axle. By analysing and testing of more than twenty concrete beams, an equation of the maximum tension stress at the end surface was established as:

$$
\sigma_{Y \max }=k \sigma_{O}
$$

where:

$\sigma_{o}{ }^{----}$The average compression stress applied on the cross-section of the beam end.

$\mathrm{k}$---- Factor of stress. Its value can be approximately represented as:

$$
k=\frac{1}{18\left(\frac{e}{h}\right)^{2}+0.25}
$$

where:

$e$---- The distance between the combined forces to the base of the concrete beam.

$h$---- The height of concrete beam at the end.

This type of crack may happen approximately at the location (the distance between the crack and bottom of the beam) C:

$$
C=\sqrt{e h}
$$

The crack resistance capability should be satisfied as:

$$
\sigma_{Y \max } \leq r f_{t},
$$

where:

$f_{t}{ }^{----}$Tensile strength of concrete.

$r$---- Plastic factor (usually use 1.7).

When utilise the above equations to calculate the tensile stress on the end of the concrete sleepers, it was found that the maximum tensile stress on the end caused by the pre-tensioning force was approximately equal to $75-90 \%$ of the permit tension stress, and equal $50 \%$ of the flexural tensile strength. This tensile stress itself cannot make the concrete sleeper crack, however, when considering the other factors such as low strength of concrete when releasing the tensile force, temperature changes and shrinking of size during the curing procedure, etc. it is very easy to develop $\sigma_{Y_{\max }} \leq f_{t}$. Hence, the longitudinal crack at sleeper end will occur. More severely, when the upper direction pulling out force applied by the wheel load at the fasteners is very high (such as high rail side of sharp curve track) and combined with the prestress forces, it can cause the longitudinal crack between the two steel shoulders to occur.

\section{METHODOLOGY OF THE NUMERICAL STUDY}

To fine tune the design works (a sample based on a good design work which is fully compliance the technical specifications), the numerical study of the concrete sleeper, relative ballast layer and their interaction was carried out by finite element method. In this study the most widely used structural finite element analysis software ANSYS was used. To obtain more accurate FEA results, the FE model was created as a three dimensional (3-D) physical 
model. In addition, material nonlinearity of concrete sleeper, steel reinforcements and interaction between the concrete sleeper and ballast layer were utilised.

\subsection{Elements for the FEA modelling}

Four types of elements were used to create the 3-D model for a newly designed "medium duty 25t axle load @ 80 km/h" concrete sleeper and the underneath thickness of 200, 250, 300 and $450 \mathrm{~mm}$ ballast layers. The SOLID65 element is used to simulate the concrete sleeper, the SOLID45 3-D 8-Node structural solid element for the ballast layer, the BEAM4 3-D beam element was used to simulate the prestressed steel tendon in the concrete sleeper, the 3D TARGE170 element and the 3D CONTA174 element to simulate the interaction surface between the concrete sleeper and ballast. The friction between concrete sleeper and ballast was simulated using a friction factor of 0.8 for the contact elements.

It has been noticed that from the 1st decade of this century, a newly developed "discrete element method (DEM)" has been used to simulate the ballast deformation behaviour, especially for the behaviour under the triaxial compression or compactness (McDowell and Li [5], Zhou et al. [6]). However, this DEM model is not suitable for this study, this is because of the ballast condition on the track is significant poorer than the new ballast, the ballast on track is mixed with very high ratio of fine particles and dusts. And, the extreme fine materials cannot be simulated by the discrete elements.

SOLID65 is used for the 3D modelling of solids with or without reinforcing bars (rebar). The solid is capable of cracking in tension and crushing in compression.

The physical FE model of the concrete sleeper and ballast layer are created using the elements mentioned above, as shown in Fig. 2. To make easy to see the details of every kind of element a FE mesh with cross section details was shown as Fig. 2 as well.

\subsection{Material nonlinearities}

To accurately represent the situation of concrete sleeper under the axle load, the nonlinearities of the concrete material and steel reinforcement were considered and simulated by means of the material nonlinearities. The nonlinear material properties of the stress and strain relationship are entered as the "multi-linear kinematic hardening" materials in the ANSYS FEA model. The material behaviour is described by a piece-wise linear stress-strain curve, starting at the origin, with positive stress and strain values (Zhang [7]).

For the reinforcement, the stress strain curve modelled in ANSYS represents an elasticperfectly plastic material identical in both tension and compression. The failure strain has been taken as 0.05 and the ultimate strength of 1.04 times the yield strength.

The material properties of the concrete sleeper are given as:

- Characteristic compressive strength $f_{C}^{\prime}=50,000 \mathrm{kPa}$

- Flexural tensile strength $f_{C f}=6010 \mathrm{kPa}$

- Finial permit compression stress $f_{P C}=22,500 \mathrm{kPa}$

- $\quad$ Finial permit tension stress $f_{P T}=2830 \mathrm{kPa}$

The following material properties have been assumed or obtained from concrete design handbook.

- Density $\rho=2.4 \mathrm{t} / \mathrm{m}^{3}$ 


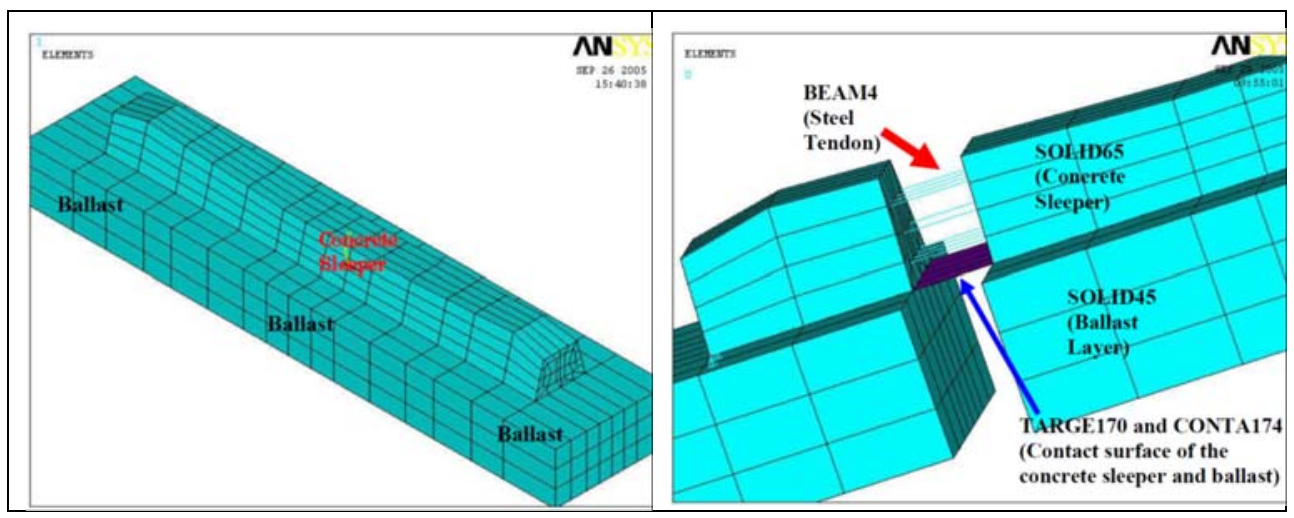

Figure 2: Finite element model of the medium duty concrete sleeper and ballast layer.

- Elastic modulus $\mathrm{E}=3.16 \times 10^{7} \mathrm{kPa}$

- Poisson's Ratio $v=0.2$

- Shear transfer coefficient* $\quad \beta_{\mathrm{t}}=0.2$

* Shear transfer coefficient was used to represent the condition of open and close of the crack. Shear in concrete can cause inclined creak across a member. Type shear transfer coefficients range from 0 to 1.0 , with 0 representing a smooth crack (Complete loss of shear transfer) and 1.0 representing a rough crack (no loss of shear transfer). This specification made for both the closed and open crack. The shear transfer coefficient for open and close crack of concrete structures was considered as 0.2 to 0.22 .

The compressive ultimate uniaxial stress-strain relationship for the concrete model was obtained using the following equations to compute the multi-linear isotropic stress-strain curve for the concrete.

$$
\varepsilon_{0}=\frac{2 f_{C}^{\prime}}{E_{C}}
$$

where:

$\varepsilon_{O^{----}}$Strain at ultimate compressive strength $f_{C}^{\prime}$. In this project, $\varepsilon_{O}=\frac{2 \times 50000}{31600000}=3.165 \times 10^{-3}$

$f_{C}^{\prime}$---- Ultimate uniaxial compressive strength $(\mathrm{kPa})$

$\mathrm{E}_{\mathrm{O}}$---- Elastic modulus of the concrete $(\mathrm{kPa})$

$$
\sigma=\frac{E_{C} \varepsilon}{1+\left(\frac{\varepsilon}{\varepsilon_{0}}\right)^{2}}
$$

where:

$\sigma$---- Stress (in plastic situation) $(\mathrm{kPa})$

$\mathrm{E}_{\mathrm{C}^{----}}$Elastic modulus of the concrete $(\mathrm{kPa})$

$\varepsilon$---- Strain 
$\varepsilon_{O}$---- Strain at ultimate compressive strength $f_{C}^{\prime}$

$$
E_{C}=\frac{\sigma}{\varepsilon}
$$

where:

$\sigma$---- Stress (in elastic situation) $(\mathrm{kPa})$

Implementation of the William and Warnke material model (for analysis of concrete structures) in ANSYS requires that different constants be defined.

To simplify the FE model and saving computational time, the ballast layers were considered as in elastic situation during the analysis.

\subsection{Applied loads}

The wheel loading details for Medium Duty type 25TAL@80km/h concrete sleeper design is dynamic wheel load. It is a combination of static wheel load and relatively dynamic influence. The dynamic wheel load on one rail is utilised as $250 \mathrm{kN}$. According to the AS 1085.14 , for concrete sleeper design the sleeper load distribution factor of approximately 0.55 for $667 \mathrm{~mm}$ spacing should be used. Hence, the vertical load is $250 \times 0.55=137.5 \mathrm{kN}$. In addition, the horizontal force equal 0.5 times vertical load, which is $68.95 \mathrm{kN}$. Therefore, the basic design load for this case is $\sqrt{(137.5)^{2}+(68.95)^{2}}=153.7 \mathrm{kN} \approx 154 \mathrm{kN}$. The $154 \mathrm{kN}$ design load was applied at 26.5 degrees to the vertical on the rail.

As the lateral load is carry out by the fastening systems, hence, only the vertical load $137.5 \mathrm{kN}$ is to be applied on the top surface of rail seat of the FEA model of concrete sleeper. The wheel load was applied to the FEA model as pressure load on the rail seat area. The angle was considered by rotating the coordination system of the nodes at the rail seat. In addition, the $2.86^{\circ}$ angle caused by the slope of rail seat was also considered.

\subsection{Boundary condition}

The concrete sleeper was laid on the ballast in the position of symmetrical in two of the three dimensions for both the geometry and loading. The boundary condition of the FEA model was defined on the assumption of no movement of the base of mid-span of concrete sleeper in the horizontal (x) direction and train traffic (z) direction. For the ballast layer, the ballast cannot be moved in five of six directions, and only the top surface is free. This was accomplished by fixing all translational degrees of freedom of the ballast outer surface areas, except for the top surface.

\section{OUTCOMES FROM THE NUMERICAL STUDY}

Based on the experience of concrete sleeper maintenance, the thickness and condition of ballast can significantly influence the performance of the concrete sleeper. Hence, within these FEA models the thickness and condition of the ballast was changed to simulate different ballast support condition to the sleepers. The ballast conditions were carried out by changing their elastic modulus value, E.

For the ballast condition study, the following FEA models were applied:

- Uniform (ideal) ballast condition;

- Centre loosing condition;

- Centre bound; 
- Centre bound combined with loosing or even gap between the sleeper and ballast at the rail seat location; and

- Different thickness of ballast layer (200mm and $250 \mathrm{~mm})$

\subsection{Uniform (ideal) ballast condition}

For the ideal ballast condition, the concrete sleeper provides good performance. From the FEA results, it is found if the ballast layer is $250 \mathrm{~mm}$ in thickness and its material properties are uniformly distributed, the maximum compression stress areas are located at the two rail seat areas, with maximum stress value of $-13032 \mathrm{kPa}$ for $\mathrm{x}$-direction and $11419 \mathrm{kPa}$ for von Mises.

The second maximum compression stress areas were located at the base of the middle of the concrete sleeper symmetry at the centre line, with maximum stress value of $-10,994 \mathrm{kPa}$ for $\mathrm{x}$-direction and $9352 \mathrm{kPa}$ for von Mises. Both the absolute stress value of $\mathrm{x}$-direction and von Mises are approximately equal to $81.9-84.4 \%$ of the value at the rail seat. In addition, both the concrete surface at the rail seat and middle bottom were under the high compression stress and are mainly in the $\mathrm{x}$-direction.

Moreover, comparing the maximum compression stress at the rail seat and centre bottom of the concrete sleeper with the final permit stresses value, which was provided by the designer at $22500 \mathrm{kPa}$, it can be found that the maximum compression stress is still $42.1 \%$ and $51.1 \%$ lower than the material limitation. Hence, it can be ensured that failure will not occur when the sleeper is under the $25 \mathrm{t}$ axial load.

Furthermore, the tension stress value both at the bottom of rail seat and top surface at the centre area are no more than $2533 \mathrm{kPa}$, which is $10.5 \%$ lower than the permit tensile stress value of $2830 \mathrm{kPa}$ as shown in Fig. 3. Hence, it probably can ensure creak will not happen when it carries the wheel load at the $250 \mathrm{~mm}$ thickness and ideal condition of ballast layer.

The deformation shape of the concrete sleeper with a scaling of $1 \times 100$ times is shown in Fig. 3. From the figure it can be found that the whole sleeper was deformed towards the $Y$ direction. The maximum deformation along the $\mathrm{Y}$ direction occurred at the area of the two rail seats, and its value is $-2.1 \mathrm{~mm}$. In addition, the middle-span area of the sleeper, which is the area around the centre line of the sleeper, it was deformed with an arch and its crown towards the up direction.

The deformation shape at the rail seats and centre line was caused by the positive bending moment applied by the wheel load and negative bending moment applied by the reaction of ballast layer at the centre area of concrete sleeper. This situation was confirmed by Australian design standard (AS 1085.14) and experiences of many countries.]

According to the Australian design standard for concrete sleeper (AS 1085.14), the maximum positive bending moment taken to occur at the rail seat for a uniform ballast support condition. The maximum negative bending moment should be taken to occur at the centre of the sleeper, under partially or totally centre-bound conditions producing tensile stress at the top and compressive stress at the underside of the sleeper. The absolute value for both positive and negative bending moment calculated by the equation offered by AS 1085.14 was $19.22 \mathrm{kN} . \mathrm{m}$.

\subsection{Deteriorated ballast condition}

Based on the experience of concrete sleeper maintenance, poor ballast condition was the major reason that could develop concrete sleeper damage and failure. The most severe ballast condition included the loosening between the sleeper and ballast below the rail seat when 


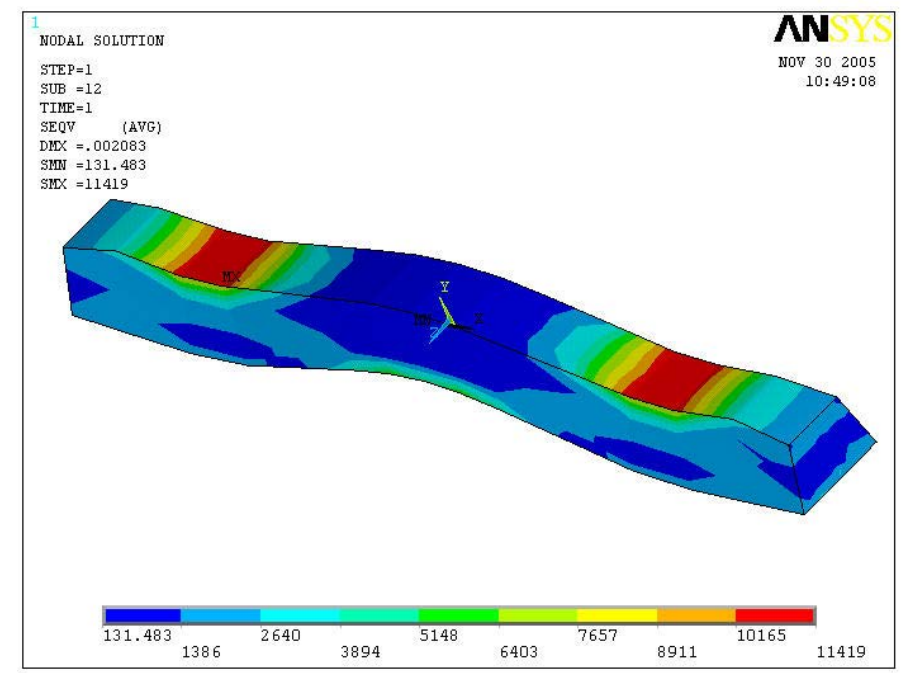

Figure 3: Deformation shape of the concrete sleeper under wheel load combined with nodal stress (von Mises, scaling $1 \times 100$ ).

maintenance is neglected, and the centre-binding caused by poor quality or neglected maintenance work as well as ballast degrading such as fouling and pumping action.

Five types of ballast conditions were modelled by means of changing the $\mathrm{E}$ value of ballast at middle-span area (the $500 \mathrm{~mm}$ width in the middle, as shown in Fig. 4) and other areas. The first condition is the uniform ballast condition $\mathrm{E}_{1}=\mathrm{E}_{2}$, in which $\mathrm{E}_{2}$ represent the elastic modulus of ballast at middle-span area and $\mathrm{E}_{1}$ is the value of other area. The second condition, is the centre loosening condition, which $\mathrm{E}_{2}=0.5 \mathrm{E}$ ( $\mathrm{E}$ is the elastic modulus value of ballast at common condition). The third condition, is the centre bound condition combined loosening condition at rail seat, in which, $\mathrm{E}_{1}=10 \% \mathrm{E}$ and $\mathrm{E}_{2}=2 \mathrm{E}$. The fourth and fifth conditions are similar to the third, but the $\mathrm{E}_{1}$ value is further smaller.

The bending moment of the concrete sleeper of these five different ballast conditions is represented by Fig. 4. (The available output data from ANSYS are the nodal/element stress and strain values. The bending moment value at a specified cross-section was obtained by summing up the moment value of selected nodes on a specified cross-section which was calculated by nodal force and relative distance).

From Fig. 4, the bending moment on the cross-section of the concrete sleeper, it is found that the maximum positive bending moment (tension on the bottom surface) was located at the rail seat areas in all of these five ballast situation. Comparing the results, the uniform ballast condition's result was about 3.2\% lower than ballast condition III (centre bound $\mathrm{E}_{1}=10 \% \mathrm{E}, \mathrm{E}_{2}=2 \mathrm{E}$ ), and $11.3 \%$ higher than the smallest value of centre bound $\mathrm{E} 1=4 \%$, $\mathrm{E} 2=2 \mathrm{E}$. Both of these five ballast conditions' results were very close. In addition, the condition I's result is very close to the result that was calculated using the equation provided by the Australian Standard AS 1085.14, which is 19.22 kN.m compared with 18.625 kN.m.

The negative bending moment occurred at the middle of the concrete sleeper, the result of ballast condition which was "centre bound" combined with loosening ballast (highly deteriorated ballast) rail seat area was much higher than the uniform ballast condition. Moreover, studying the three centre-bound ballast conditions, it was found that the ballast 


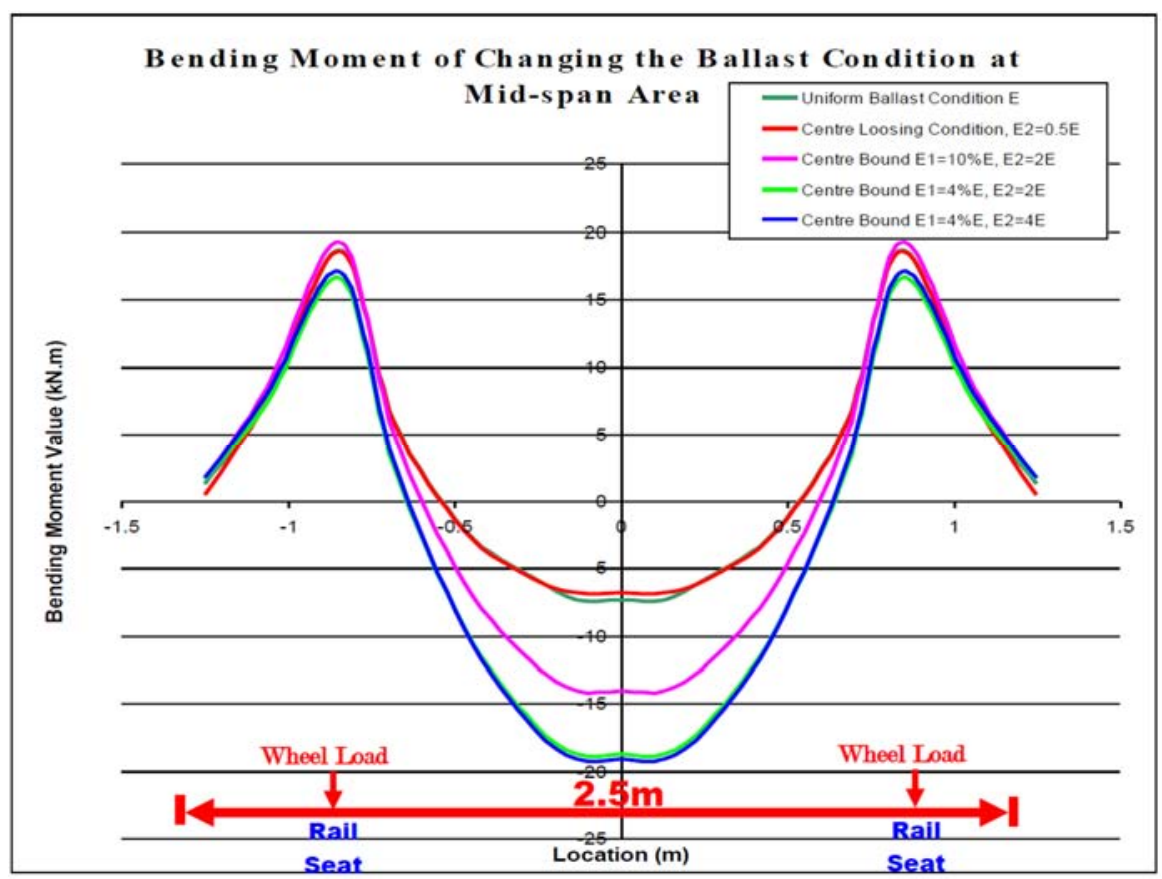

Figure 4: The bending moment on the cross-section of the concrete sleeper.

condition at the rail seat location can significantly influence the negative bending moment at the middle of the concrete sleeper. Changing the E value at the middle span area also can influence the negative bending moment results, but its results cannot be compared with changing the condition of ballast at the rail seat.

\subsection{Deflection shape of the concrete sleeper}

To understand the deformation shape of the concrete sleeper lay on uniform and centre bound ballast layer, the nodal vertical displacement of the FEA sleeper models under the wheel load were represented in Fig. 5.

Studying the diagram of the vertical displacement of the sleeper under the wheel load along the longitudinal direction of the sleeper, some findings were obtained.

Firstly, the deformation shapes of the concrete sleeper against the two ballast conditions were similar. The vertical displacements are changing like "Arch" from one end to another end. The two lowest points (maximum negative value) were found at the two rail seat locations, and the middle area was deformed towards the upper direction that caused the ballast reaction at that area.

Secondly, the vertical displacements were significantly changing with the different ballast condition. For uniform ballast condition the concrete sleeper provided good performance in deflection under the 25-ton axle load. Its maximum vertical displacement at rail seat and centre were $-2.06 \mathrm{~mm}$ and $-0.72 \mathrm{~mm}$, respectively. Moreover, the deformation at the centrebound and loosing/gap ballast at rail seat condition is also well matched with the damage conditions which were happened on the track. 


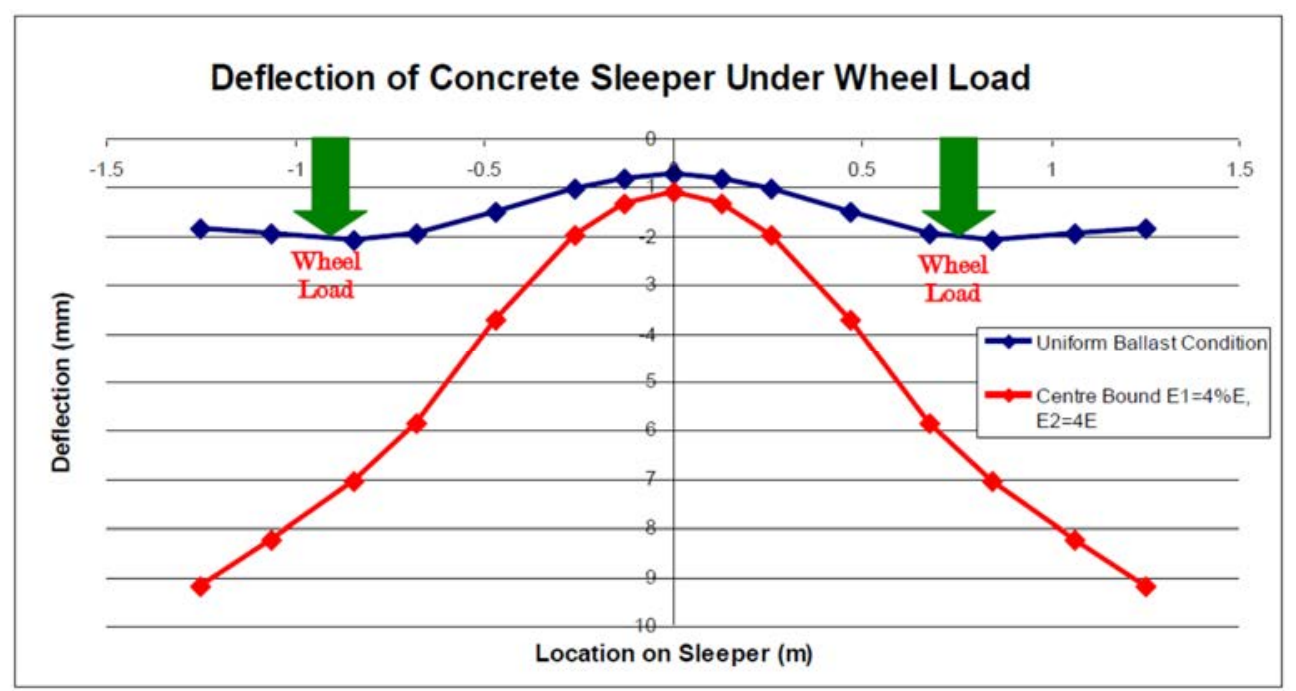

Figure 5: The Y-direction displacement of the concrete sleeper under the wheel load.

\subsection{X-direction bending stress}

X-direction bending stress distribution of the newly designed concrete sleeper is good. Based on design experience, the predominant bending stress of the concrete sleeper under the wheel load is the $\mathrm{x}$-direction stress along the longitudinal direction of the sleeper. The $\mathrm{x}$-direction stress was the most important reason that could develop the concrete sleeper crack or crush under the load. The x-direction stress of the top of the concrete sleeper along the longitudinal centre line at three ballast conditions were plotted as the diagrams in Fig. 6.

The concrete sleeper represents the compression stress in the x-direction both at the top and bottom surface. The maximum stress at the top surface was at the rail seat areas, while at the bottom of sleeper it was located at the middle area. For the uniform ballast condition, the maximum stress value at the top surface was about $16 \%$ higher than the bottom surface. However, if the ballast was in the centre-bound and rail seat loosening condition, the compressive stress at the centre of sleeper base can be more than two times higher than the uniform ballast condition.

Moreover, checking the tensile stress at the top surface of the middle $500 \mathrm{~mm}$ area of the concrete sleeper, it found that even in the centre-bound condition the positive x-direction stress value is still very low ( $\left.\sigma_{\max }=168 \mathrm{kPa}\right)$.

Hence, the horizontal and mesh cracks on the top surface of middle area of concrete sleeper can be avoided. However, with the uniform ballast condition, the maximum $\mathrm{x}$ direction tensile stresses $2528 \mathrm{kPa}$ were found at the bottom surface of the two end areas.

Confirmed by the tests conducted in China in 1990s, it is believed that this sort of higher $\mathrm{x}$-direction stress have relationship with the cracks on the surface of sleeper base at its two ends. This situation will more serious when it is combined with other hazard track conditions, such as fouling, sharp curve, etc. 


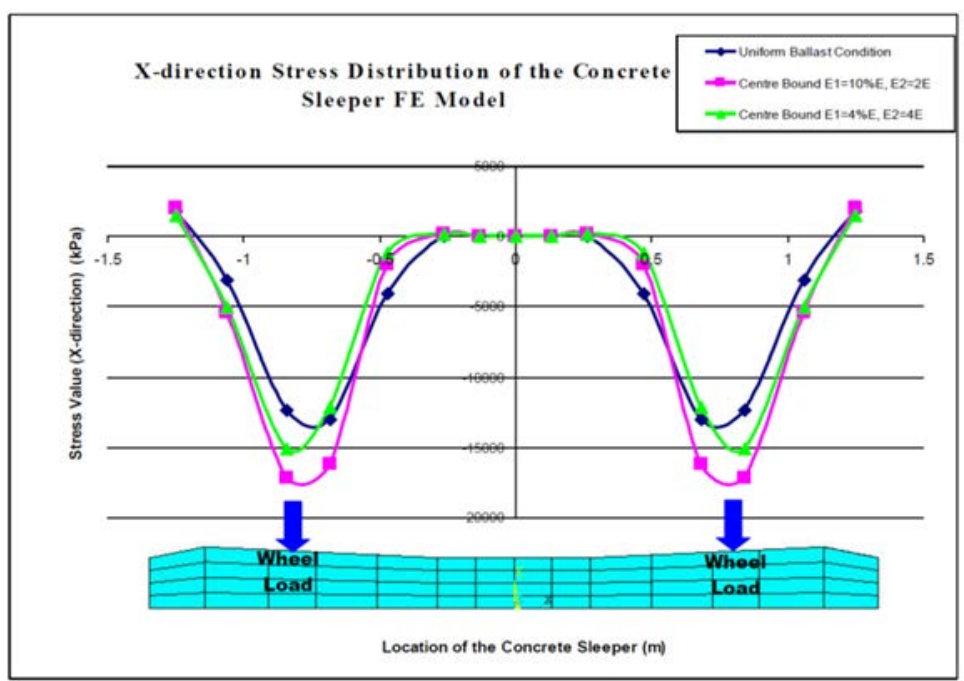

Figure 6: The nodal stress (x-direction) of the concrete sleeper top.

\subsection{Ballast pressure}

Based on the FEA results, the maximum pressure results were changing with the ballast conditions that mentioned above. With the uniform ballast condition, the locations of the maximum ballast pressure of these three conditions were all found at the area where under the rail seat. The lowest values were located at the middle areas. It can be seen that the maximum ballast pressure for uniform ballast condition (ideal condition and also AS 1085 design condition) is $528 \mathrm{kPa}$, which is nearly $5.6 \%$ higher than the requirement $500 \mathrm{kPa}$. These results were confirmed by the calculation based on the equation suggested by Australian Standard AS 1085.14. The method that can improve the original design is to increase the width of concrete sleeper from current $290 \mathrm{~mm}$ to $300-305 \mathrm{~mm}$.

\section{CONCLUSIONS AND RECOMMENDATIONS}

Considering environments that the concrete sleepers to be installed will include many of the arduous locations, such as sharp curves, a sleeper must withstand various types of loads, weather conditions, without losing its properties during its service life. Hence, some special suggestions of improvement are provided.

\section{Type of reinforcement}

There are many evidences have shown that the pre-tensioned multi-ply steel wires/strands may cause more serious longitudinal crack at sleeper ends and between the two fasteners. As a result, the higher diameter $(6 \mathrm{~mm}-10 \mathrm{~mm})$ single reinforcing steel tendons are recommended. For the further study, a new FEA model will be created to undertake the compare study of the performances between the single reinforcement and multi-ply steel wires within the concrete sleeper. However, many challenges and technical difficulties are to be solved before the numerical study can be carried out.

Avoiding longitudinal cracks between the pair of steel shoulders

Another important reason that can develop this type of sleeper failure is the higher tensile stress at the steel shoulder (fasteners) area (Ma et al. [8]). This situation will be severer when concrete sleepers are installed in sharp curves where this stress may combined with the very 


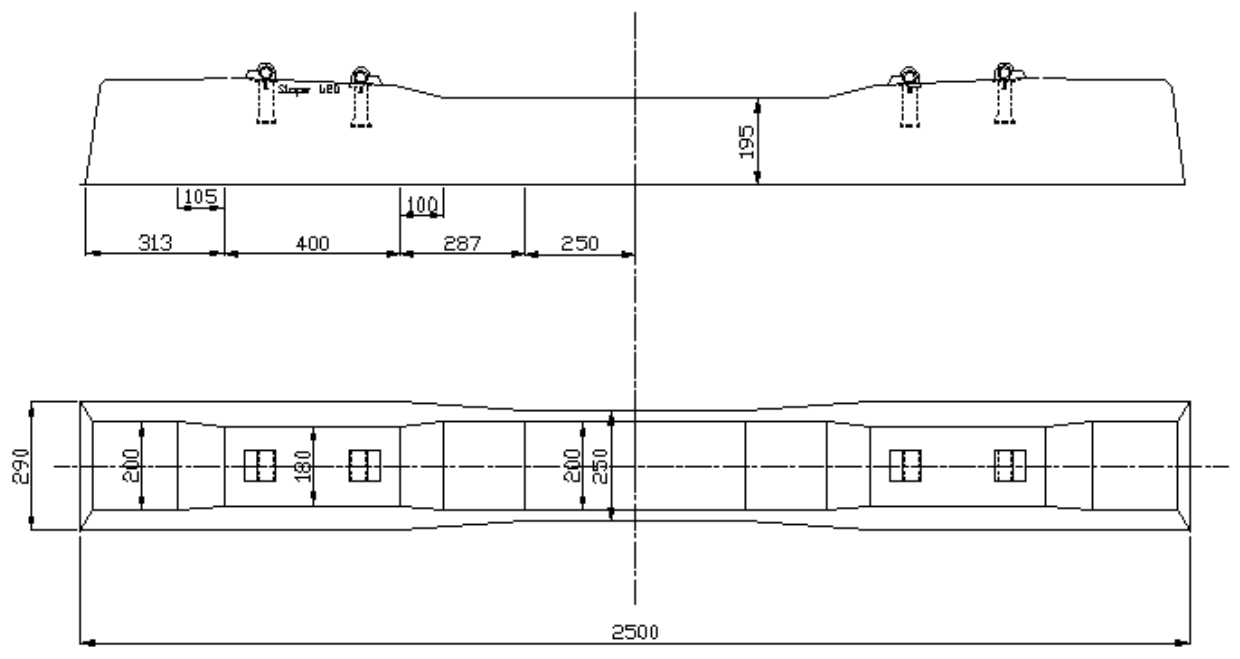

Figure 7: Example of the sleeper shape.

high uplift pulling out force that is applied by the wheel load at the fasteners. A potential solution is by applying web distributed steel reinforcement at the sleeper end and spiral tendons around the fasteners location.

Longitudinal shape of concrete sleeper

The optimization of the longitudinal shape of the concrete sleeper is a procedure to make a balance of the required geometry at different areas and cross-sections. Both safety and cost should be considered. Based on FEA results and concrete sleeper design experience, the middle $500 \mathrm{~mm}$ length of sleeper can be designed to apply narrow width and lower height than the rail seat to reduce resource and weight of sleeper. A good shape of a $2.5 \mathrm{~m}$ length middle duty concrete sleeper is as shown in Fig. 7.

\section{REFERENCES}

[1] Australian Standard, Australian Standard: Railway track material. Part 14: Prestressed concrete sleepers. AS 1085.14. 2003.

[2] Gustavson, R., Static and dynamic finite element analysis of concrete sleeper. Thesis for the Degree of Licentiate of Engineering, Division of Concrete Structures, Department of Structural Engineering, Chalmers University of Technology, Goteborg, Sweden, 2000.

[3] Vincent, G., Modal analysis and numerical modeling of a concrete railway sleeper. Thesis for the Degree of Licentiate of Engineering, Division of Concrete Structures, Department of Structural Engineering, Chalmers University of Technology, Goteborg, Sweden, Jun. 2001.

[4] Wang, J. \& Bai, L., The crack and structural durability of presstress railway concrete sleeper. Concrete and Concrete Productions, Apr. 2004. (in Chinese.)

[5] McDowell, G. \& Li, H., Discrete element modelling of scaled railway ballast under triaxial conditions. Granular matter, 18(66), 2016. DOI 10.1007/s10035-016-0663-8. 
[6] Zhou, T., Hu, B., Sun, J. \& Liu., Z, Discrete element method simulation of railway ballast compactness during tamping process. The Open Electrical \& Electronic Engineering Journal, 7, pp. 103-109, 2013.

[7] Zhang, R., The behavior of long span deep-corrugated reinforced steel box culverts. Thesis of Master of Engineering, Faculty of Engineering, University of Technology, Sydney, Sept., Australia., pp. 26-34, 2004.

[8] Ma, C., Dou, H. \& Niu, H., Measures for prevention of longitudinal cracks near bolt hole of concrete sleeper. Railway Engineering, 4, 2004. (in Chinese.) 